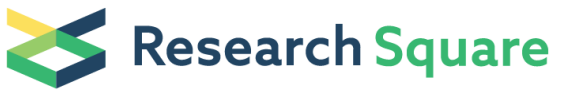 \\ Preprints are preliminary reports that have not undergone peer review. \\ They should not be considered conclusive, used to inform clinical practice, or referenced by the media as validated information.
}

\section{Correlation Between the TERT rs2736100 A/C Polymorphism and Susceptibility to Interstitial Lung Diseases: An Ethnicity-Based Meta- Analysis Study}

Beibei Cui

Sichuan University West China Hospital

Yuehong Chen

Sichuan University West China Hospital

Xinyue Ma

West China Hospital of Medicine: Sichuan University West China Hospital

Geng Yin

Sichuan University West China Hospital

Qibing Xie ( $\nabla$ xieqibing1971@163.com)

Sichuan University West China Hospital https://orcid.org/0000-0002-6727-1839

\section{Research Article}

Keywords: TERT, Rs2736100, Interstitial lung disease, Connective tissue disease, Idiopathic pulmonary fibrosis

Posted Date: March 8th, 2022

DOI: https://doi.org/10.21203/rs.3.rs-1414064/v1

License: (-) (1) This work is licensed under a Creative Commons Attribution 4.0 International License. Read Full License 


\section{Abstract}

Objective: This meta-analysis aimed to investigate the correlation between the TERT rs $2736100 \mathrm{~A} / \mathrm{C}$ polymorphism and susceptibility to interstitial lung diseases (ILD).

Methods: The systematic review was registered in PROSPERO. OVID MEDLINE, OVID EMBASE, and Web of Science electronic databases were searched. The meta-analysis effect size was estimated using either incidence with $95 \%$ confidence intervals (Cls) or odds ratio (OR). A funnel plot was used to assess the risk of publication bias.

Results $₫ A$ total of 12 eligible studies were finally analyzed, involving 5272 cases and 14767 controls. The frequency of rs $2736100 \_C$ was lower in patients with ILD (38.4\% vs $47.3 \%)$. Asian population had a lower rs 2736100 C frequency than European population (32.8\% vs $40 \%)$. A significant association between rs2736100_A and ILD was identified ( $p<0.00001$, OR 1.36, 95\% Cl 1.24-1.48). The incidence of ILD increased with rs2736100_A in both European population ( $\mathrm{p}<0.00001$, OR 1.34, 95\% Cl 1.27-1.41) and Asian population ( $\mathrm{p}=0.008,0 \mathrm{R} 1.42,95 \% \mathrm{Cl} 1.10-1.85)$. Among six idiopathic pulmonary fibrosis (IPF) studies, rs2736100_A was associated with the incidence of IPF ( $p<0.00001,0 R$ 1.53, 95\% Cl 1.27-1.85). All genetic models, including recessive, dominant, and additive models, showed increased risk of IPF was associated with rs2736100_A. Rs2736100_A was not associated with the incidence ofconnective tissue disease associated ILD (CTD-ILD) ( $p=0.75,0 R 1.07,95 \% \mathrm{Cl} 0.71-1.61)$.

Conclusion: This study demonstrated the frequency of rs2736100_A was lower in Asian population. We confirmed the TERT rs2736100 A/C polymorphism was a strong risk factor for IPF, but not for CTD-ILD. Whether the incidence of CTD-ILD increases with the TERT rs2736100 A/C polymorphism needs to be evaluated in the future.

\section{Introduction}

Interstitial lung disease (ILD) is characterized by fibrosis and inflammation in the pulmonary parenchyma. ILD encompasses a large, heterogeneous group of entities, such as idiopathic interstitial pneumonias (IIP), environmental exposure-related ILD, connective tissue disease associated ILD (CTD-ILD), pulmonary sarcoidosis and smoking-related ILD [1]. Of note, a progressive fibrosing phenotype that represented by idiopathic pulmonary fibrosis (IPF) leads to aggressive fibrosing, decline in lung function, worsening symptoms and poor prognosis [2]. Besides of IPF, this phenotype can also be observed in CTD-ILD, fibrotic non-specific interstitial pneumonia, chronic hypersensitivity pneumonitis (HP) and asbestos-induced lung fibrosis [3-4].

Emerging evidences suggest that the incidence of ILD increases with telomere abnormalities and mutations in telomere related genes (TRGs), including telomerase reverse transcriptase (TERT) catalytic subunit and the telomerase RNA component (TERC), poly(A)-specific ribonuclease (PARN), regulator of telomere elongation helicase 1(RTEL1), and TERTF1 interacting nuclear factor 2 (TINF2) (Diaz de Leon, et al. 2010; Newton et al., 2016; Borie et al. 2019). So far, TERT mutations have been pointed to be associated with familial IPF, sporadic IPF, NSIP, desquamative interstitial pneumonia, CTD-ILD, and chronic hypersensitivity pneumonitis [5-7].

\section{Rationale}

Rs2736100 polymorphism, an important single nucleotide polymorphism in the intron region of TERT, has been associated with ILD in several genome wide association studies (GWAS), but the results of these studies were conflicting. Some of studies found significant associations between rs2736100 polymorphism and IIP patients, whereas others did not [8-11]. The rs 2736100 risk allele was associated with shorter telomere length in IPF patients, but not in non-IPF patients [6,12]. Furthermore, the role of the TERT rs 2736100 polymorphism seems to differ substantially between populations [11].

\section{Objectives}

Therefore, we conducted this meta-analysis to investigate the incidence and risk of the TERT rs2736100 polymorphism in patients with ILD and to better illuminate the relation between the TERT rs2736100 polymorphism and ILD.

\section{Materials And Methods}

\section{Ethical compliance}

This study does not involve with ethics as it is a systematic review and meta-analysis.

\section{Study registration}

The systematic review was registered in PROSPERO (CRD42022303887). We followed the Preferred Reporting Items for Systemic Reviews and Meta-Analyses 2009 statement.

\section{Search Strategy}


To perform systematic retrieval, we searched the electronic databases OVID MEDLINE, OVID EMBASE, and Web of Science using a Medical Subject Headings term and a keyword on Jan 30, 2022. The terms used in searching were as follow: The search terms were "interstitial lung diseases", "ILD", "Genetic Variation", "variant", "gene polymorphism", "TERT", "telomerase reverse transcriptase" . The detailed search strategy is provided in the supplemental file. The duplication were removed. The references of retrieved publications were manually filtered for potentially relevant articles.

\section{Inclusion criteria and exclusion criteria}

Inclusion criteria were as follow: (a) contained original data; (b) provide adequate data to calculate odds ratios (ORs) and $95 \%$ confidence intervals (Cls). Exclusion criteria were as follow: (a) contained overlapping data; (b) family member had been studied because the analyses were based on linkage considerations. (c) English texts were not available. (d) abstracts, reviews, comments and conference presentations.

\section{Data extraction and quality assessment}

Retrieved studies were filtered by titles and abstracts based on our study selection criteria. The full texts of the remaining studies from the first screening were downloaded for further screening based on the study eligibility criteria. Two authors independently screened the studies, and any disagreement was resolved via discussion or adjudication by a third reviewer, if necessary.

Two authors independently collected data on the first author's family name, year of publication, country, study design, ethnicity, the number of participants, classification of ILD, alleles frequency, genotypic distribution of rs2736100. If a study contained several independent groups, the groups would be listed respectively.

The methodological quality assessment of included studies was conducted using the Newcastle-Ottawa quality assessment scale (NOS). A total of three domains - selection, comparability, and exposure - with eight numbered items yielded the highest total score of 9 . For selection and exposure, each of seven numbered items was scored as 1 if the answer was yes, while for comparability, a maximum score of 2 was given for a numbered item. Studies with a score $\geq 6$ were considered high-quality studies. Two authors performed the methodological quality assessment, and any disagreement was resolved via discussion or adjudication by a third reviewer, if necessary.

\section{Statistical analysis}

We performed data analyses using RevMan software (version 5.4). The probability value ( $p$ value) of Hardy-Weinberg equilibrium (HWE) was calculated. (https://ihg.gsf.de/cgi-bin/hw/hwa1.pl). Meta-analyses were performed using 4 models, including allelic contrast, additive, recessive and dominant. Subgroup analysis was performed according to ethnicity and classification of ILD.

The effect size of the meta-analysis was estimated by incidence with $95 \%$ confidence intervals (Cls) and odds ratio (OR). We assessed clinical diversity across studies through statistical heterogeneity using $\mathrm{I}^{2}$ and $\mathrm{p}$-values. $\mathrm{I}^{2}$ values of $25 \%, 50 \%$, and $75 \%$ represented low, moderate, and high heterogeneity, respectively. Fixed-effect models (FEMs) were used for synthetic analyses. A random effect model was applied when heterogeneity was over $50 \%$ or $\mathrm{P}<0.05$. Sensitivity analysis was performed by excluding studies one by one to identify the potential source of heterogeneity. We assessed the risk of publication bias via funnel plot. The power of each study on assessing the association between rs2736100 and ILD was conducted via G*Power (convention $w=0.1$, http://www.gpower.hhu.de/).

\section{Results}

\section{Literature Search and Study Characterisitc}

A total of 831 studies were retrieved via electronic and manual searching, with 17 selected articles for further evaluation based on title and abstract details. Seven articles were excluded due to incomplete data. Among the 10 included articles, two studies contained complete data from two independent groups, so we listed them respectively. A total of 12 eligible studies were finally analyzed, involving 5272 cases and 14767 controls. The details are shown in Fig. 1.

A total of 12 eligible studies were finally analyzed, involving 5272 cases and 14767 controls. The studies included 5 IPF studies, 2 CTD-ILD studies, 1 environment-exposured ILD study, 3 IIP studies (one study contains specific data of IPF and non-IPF groups), 1 familial ILD study. All of the 12 eligible studies were case-control studies. Seven studies involved European populations, whereas five studies involved Asian population. The characteristics of eligible studies are shown in Table 1. The power of eligible studies varies from 37.2-99.9\%. 
Table 1

Characterisitics of individual studies included in the mata-analysis

\begin{tabular}{|c|c|c|c|c|c|c|c|c|c|c|c|c|c|}
\hline \multirow[t]{2}{*}{ References } & \multirow[t]{2}{*}{ Year } & \multirow[t]{2}{*}{ Country } & \multirow[t]{2}{*}{ Ethnicity } & \multirow[t]{2}{*}{ Disease } & \multicolumn{2}{|c|}{ Numbers } & \multicolumn{2}{|c|}{$\begin{array}{l}\text { Minor alleles } \\
(\%)\end{array}$} & \multicolumn{4}{|c|}{ Allele association } & \multirow[t]{2}{*}{ Power(\%) ${ }^{*}$} \\
\hline & & & & & Case & Control & Case & Control & OR & $95 \%$ & & $p$ value & \\
\hline $\begin{array}{l}\text { Guzmán- } \\
\text { Vargas }^{[20]}\end{array}$ & 2021 & Mexico & European & IPF & 93 & 174 & 25 & 38.9 & 0.52 & 0.34 & 0.8 & 0.0028 & 37.2 \\
\hline Jonsson $^{[10]}$ & 2021 & Sweden & European & RA-ILD & 60 & 2350 & 39.2 & 45.9 & 0.76 & 0.52 & 1.1 & 0.1447 & 99.8 \\
\hline $\begin{array}{l}\text { Arimura- } \\
\text { Omori }^{[21]}\end{array}$ & 2020 & Japan & Asian & IPF & 155 & 379 & 28.7 & 41.3 & 0.57 & 0.43 & 0.76 & 0.0001 & 63.7 \\
\hline Kawasaki $^{[16]}$ & 2020 & Japan & Asian & $\begin{array}{l}\text { AAV- } \\
\text { ILD }\end{array}$ & 176 & 216 & 34.7 & 31.6 & 1.15 & 0.8 & 1.66 & 0.4468 & 50.8 \\
\hline Yuan [22] & 2020 & China & Asian & CWP & 645 & 626 & 38.4 & 42.7 & 0.84 & 0.72 & 0.98 & 0.031 & 94.6 \\
\hline Mathai ${ }^{[23]}$ & 2019 & USA & European & $\mathrm{PF}$ & 77 & 417 & 44.8 & 45 & 0.99 & 0.7 & 1.4 & 0.971 & 60.3 \\
\hline Dressen $^{[12]}$ & 2018 & USA & European & IPF & 1510 & 1874 & 42.7 & 49 & 0.78 & 0.7 & 0.86 & $\begin{array}{l}<.00001 \\
0 .\end{array}$ & 99.9 \\
\hline $\mathrm{Wei}^{[14]}$ & 2014 & USA & European & ILD & 277 & 689 & 43.8 & 50.1 & 0.78 & 0.63 & 0.96 & 0.0198 & 87.5 \\
\hline $\begin{array}{l}\text { Fingerlin - } \\
1^{[8]}\end{array}$ & 2013 & USA & European & IIP & 1616 & 4683 & 43 & 51.1 & 0.72 & 0.66 & 0.78 & $\hat{0}_{0.00001}$ & 99.9 \\
\hline $\begin{array}{l}\text { Fingerlin - } \\
2^{[8]}\end{array}$ & 2013 & USA & European & IIP & 876 & 1890 & 42.9 & 50.1 & 0.75 & 0.67 & 0.84 & $\begin{array}{l}<.00001 \\
0 .\end{array}$ & 99.9 \\
\hline $\begin{array}{l}\text { Mushiroda } \\
-1^{[9]}\end{array}$ & 2008 & Japan & Asian & IPF & 159 & 934 & 27 & 40.9 & 0.54 & 0.41 & 0.7 & $\begin{array}{l}< \\
0.00001\end{array}$ & 91.1 \\
\hline $\begin{array}{l}\text { Mushiroda } \\
-2^{[9]}\end{array}$ & 2008 & Japan & Asian & IPF & 83 & 535 & 28.9 & 41 & 0.58 & 0.41 & 0.84 & 0.0032 & 70.1 \\
\hline $\begin{array}{l}\text { OR, odds ratic } \\
\text { workers' pneu } \\
0.05 \text { significa }\end{array}$ & $\begin{array}{l}\text {, cor } \\
\text { coni } \\
\text { leve }\end{array}$ & $\begin{array}{l}\text { nce inte } \\
; \text { PF, pulr }\end{array}$ & $\begin{array}{l}\text { l; IPF, idiop } \\
\text { nary fibros }\end{array}$ & $\begin{array}{l}\text { ic pulm } \\
\text { LD, inter }\end{array}$ & $\begin{array}{l}\text { ary fib } \\
\text { ial lur }\end{array}$ & $\begin{array}{l}\text { is; RA, } \\
\text { diseas }\end{array}$ & $\begin{array}{l}\text { umat } \\
\text { IP, idi }\end{array}$ & $\begin{array}{l}\text { arthritis } \\
\text { thic inte }\end{array}$ & $\begin{array}{l}\text { AV, Al } \\
\text { titial }\end{array}$ & $\begin{array}{l}\text {-ass } \\
\text { umo }\end{array}$ & ated & $\begin{array}{l}\text { sculitis; CV } \\
\text { lention w }\end{array}$ & $\begin{array}{l}\text { coal } \\
.1 \text { at a }\end{array}$ \\
\hline
\end{tabular}

\section{Methodological quality}

Of the ten studies, 8 studies had a NOS score of $\geq 6$, indicating high study quality, while 2 studies were rated as being of low quality (NOS score < 6). The NOS scores are shown in supplemental tables $\mathbf{S 1 .}$

\section{Allele frequency of the TERT rs2736100 A/C polymorphism}

Compared with controls, the frequency of rs2736100_C was lower in patients with ILD (38.4\% vs 47.3\%). Asian population had a lower rs2736100_C frequency than European population (32.8\% vs 40\%). The details are shown in Table 2.

Table 2

MAF of the TERT rs2736100 polymorphism

\begin{tabular}{|llllll|}
\hline Population & \multirow{2}{*}{ No. of studies } & \multicolumn{2}{l}{ Numbers } & MAF & \\
\cline { 3 - 6 } & & ILD & Control & ILD & Control \\
\hline European & 7 & 4509 & 12077 & 0.40 & 0.49 \\
\hline Asian & 5 & 1218 & 2690 & 0.34 & 0.4 \\
\hline Total & 12 & 5727 & 14767 & 0.38 & 0.47 \\
\hline MAF, minor allele frequency; ILD, interstitial lung diseases. & \\
\hline
\end{tabular}

\section{Meta-analysis of the TERT rs2736100 A/C polymorphism and susceptibility to ILD}

A significant association between rs2736100_A and ILD was identified ( $p<0.00001$, OR 1.36, 95\% Cl 1.24-1.48). Ethnicity-specific meta-analysis demonstrated the incidence of ILD increased with rs2736100_A in both European population ( $p<0.00001,0 R 1.34,95 \%$ Cl 1.27-1.41) and Asian 
population ( $p=0.008$, OR 1.42, 95\% Cl 1.10-1.85). All genetic models, including recessive, dominant, and additive models, showed increased ILD risk was associated with rs2736100_A in both European population and Asian population. The details are shown in Table 3 and Fig. 2.

Table 3

Analysis of the association between the TERT rs2736100 polymorphism and ILD

\begin{tabular}{|c|c|c|c|c|c|c|c|c|}
\hline \multirow[t]{2}{*}{ Polymorphism } & \multirow[t]{2}{*}{ Population } & \multirow[t]{2}{*}{ No. of studies } & \multicolumn{3}{|c|}{ Test of association } & \multicolumn{3}{|c|}{ Test of heterogeneity } \\
\hline & & & OR & $95 \% \mathrm{Cl}$ & p value & Model & $p$ value & $\mathrm{I}^{2}(\%)$ \\
\hline \multirow[t]{3}{*}{ A vs. C allele } & Overall & 12 & 1.36 & $1.24-1.48$ & $<0.00001$ & $\mathrm{RE}$ & 0.008 & 57 \\
\hline & European & 7 & 1.34 & $1.27-1.41$ & $<0.00001$ & FE & 0.35 & 11 \\
\hline & Asian & 5 & 1.42 & $1.10-1.85$ & 0.008 & RE & 0.001 & 78 \\
\hline \multirow[t]{3}{*}{$A A+A C$ vs. $C C$ (dominant) } & Overall & 9 & 1.42 & $1.31-1.54$ & $<0.00001$ & FE & 0.06 & 47 \\
\hline & European & 5 & 1.41 & $1.29-1.53$ & $<0.00001$ & $\mathrm{FE}$ & 0.1 & 49 \\
\hline & Asian & 4 & 1.65 & $1.10-2.48$ & 0.02 & $\mathrm{RE}$ & 0.07 & 58 \\
\hline \multirow[t]{3}{*}{ AA vs. $A C+C C$ (recessive) } & Overall & 9 & 0.65 & $0.57-0.74$ & $<0.00001$ & RE & 0.004 & 64 \\
\hline & European & 5 & 0.7 & $0.60-0.82$ & $<0.00001$ & $\mathrm{RE}$ & 0.01 & 68 \\
\hline & Asian & 4 & 0.56 & $0.43-0.73$ & $<0.00001$ & $\mathrm{RE}$ & 0.06 & 59 \\
\hline \multirow[t]{3}{*}{ AA vs. CC (additive) } & Overall & 9 & 1.77 & $1.51-2.07$ & $<0.00001$ & $\mathrm{RE}$ & 0.04 & 50 \\
\hline & European & 5 & 1.63 & $1.47-1.80$ & $<0.00001$ & FE & 0.21 & 32 \\
\hline & Asian & 4 & 2.2 & $135-3.59$ & $<0.00001$ & $\mathrm{RE}$ & 0.03 & 68 \\
\hline \multirow[t]{3}{*}{ AC vs. CC (additive) } & Overall & 9 & 1.25 & $1.14-1.36$ & $<0.00001$ & FE & 0.10 & 40 \\
\hline & European & 5 & 1.33 & $1.12-1.58$ & $<0.00001$ & $\mathrm{RE}$ & 0.04 & 59 \\
\hline & Asian & 4 & 1.19 & $0.94-1.51$ & 0.16 & $\mathrm{FE}$ & 0.34 & 10 \\
\hline
\end{tabular}

Meta-analysis of the TERT rs2736100 A/C polymorphism and susceptibility to IPF

Among all of six IPF studies, rs2736100_A was associated with the incidence of IPF ( $<<0.00001$, OR 1.53, 95\% CI 1.27-1.85). Significant increase of IPF risk was found for rs2736100_A in both European population ( $p=0.02$, OR 1.33, 95\% Cl 1.06-1.67) and Asian population ( $p<0.00001,0 R$ $1.79,95 \% \mathrm{Cl} 1.51-2.12)$. All genetic models, including recessive, dominant, and additive models, showed increased IPF risk was associated with rs2736100_A in both European population and Asian population. The details are shown in Table 4 and Fig. 2. 
Table 4

Analysis of the association between the TERT rs2736100 polymorphism and IPF

\begin{tabular}{|c|c|c|c|c|c|c|c|c|}
\hline \multirow[t]{2}{*}{ Polymorphism } & \multirow[t]{2}{*}{ Population } & \multirow[t]{2}{*}{ No. of studies } & \multicolumn{3}{|c|}{ Test of association } & \multicolumn{3}{|c|}{ Test of heterogeneity } \\
\hline & & & OR & $95 \% \mathrm{Cl}$ & $\mathrm{p}$ value & Model & $p$ value & $\mathrm{I}^{2}(\%)$ \\
\hline \multirow[t]{3}{*}{ A vs. C allele } & Overall & 6 & 1.53 & $1.27-1.85$ & $<0.00001$ & $\mathrm{RE}$ & 0.010 & 67 \\
\hline & European & 3 & 1.33 & $1.06-1.67$ & 0.02 & $\mathrm{RE}$ & 0.11 & 55 \\
\hline & Asian & 3 & 1.79 & $1.51-2.12$ & $<0.00001$ & $\mathrm{FE}$ & 0.91 & 0 \\
\hline \multirow[t]{3}{*}{$A A+A C$ vs. $C C$ (dominant) } & Overall & 6 & 1.41 & $1.24-1.61$ & $<0.00001$ & $\mathrm{FE}$ & 0.09 & 48 \\
\hline & European & 3 & 1.32 & $1.15-1.52$ & $<0.00001$ & $\mathrm{FE}$ & 0.15 & 47 \\
\hline & Asian & 3 & 2.09 & $1.45-3.00$ & $<0.00001$ & FE & 0.72 & 0 \\
\hline \multirow[t]{3}{*}{$A A$ vs. $A C+C C$ (recessive) } & Overall & 6 & 0.61 & $0.47-0.80$ & 0.0004 & RE & 0.004 & 71 \\
\hline & European & 3 & 0.78 & $0.69-0.87$ & $<0.00001$ & $\mathrm{FE}$ & 0.19 & 40 \\
\hline & Asian & 3 & 0.48 & $0.39-0.60$ & $<0.00001$ & FE & 0.95 & 0 \\
\hline \multirow[t]{3}{*}{ AA vs. CC (additive) } & Overall & 6 & 2.12 & $1.43-3.12$ & 0.0002 & $\mathrm{RE}$ & 0.01 & 65 \\
\hline & European & 3 & 1.61 & $1.02-2.54$ & 0.04 & RE & 0.13 & 51 \\
\hline & Asian & 3 & 2.85 & $1.95-4.16$ & $<0.00001$ & $\mathrm{FE}$ & 0.83 & 0 \\
\hline \multirow[t]{3}{*}{ AC vs. CC (additive) } & Overall & 6 & 1.26 & $1.10-1.45$ & 0.0009 & $\mathrm{FE}$ & 0.46 & 0 \\
\hline & European & 3 & 1.23 & $1.06-1.42$ & 0.007 & $\mathrm{FE}$ & 0.22 & 34 \\
\hline & Asian & 3 & 1.52 & $1.04-2.24$ & 0.03 & FE & 0.73 & 0 \\
\hline
\end{tabular}

OR, odds ratio; Cl, confidence interval; RE, R random effects model; FE, F fixed effects model; IPF, idiopathic interstitial pneumonia.

\section{The TERT rs2736100 A/C polymorphism and susceptibility to other ILD}

For meta-analysis of the TERT rs 2736100 A/C polymorphism and susceptibility to other ILD, we analyzed 2 CTD-ILD studies, 1 other ILD study and 1 CWP study.The association between rs2736100_A and ILD patients with other ILD was identified $(p=0.002,0 R 1.21,95 \%$ Cl 1.07-1.36). Rs2736100_A was not associated with the incidence of CTD-ILD ( $p=0.75$, OR 1.07, 95\% Cl 0.71-1.61). The details are shown in Table 5 and Fig. 2.

Table 5

Analysis of the association between the TERT rs2736100 polymorphism and other ILD

\begin{tabular}{|c|c|c|c|c|c|c|c|c|c|c|}
\hline \multirow[t]{2}{*}{ Polymorphism } & \multirow[t]{2}{*}{ Population } & \multirow[t]{2}{*}{ No. of studies } & \multicolumn{2}{|c|}{ Numbers } & \multicolumn{3}{|c|}{ Test of association } & \multicolumn{3}{|c|}{ Test of heterogeneity } \\
\hline & & & Case & Control & OR & $95 \% \mathrm{Cl}$ & $p$ value & Model & $p$ value & $\mathrm{I}^{2}(\%)$ \\
\hline \multirow[t]{2}{*}{ A vs. C allele } & Overall & 4 & 318 & 3183 & 1.2 & $0.89-1.62$ & 0.23 & RE & 0.08 & 60 \\
\hline & CTD-ILD & 2 & 175 & 2497 & 1.07 & $0.71-1.61$ & 0.75 & $\mathrm{RE}$ & 0.12 & 59 \\
\hline
\end{tabular}

OR, odds ratio; Cl, confidence interval; RE, R random effects model; FE, F fixed effects model; CTD-ILD, connective tissue disease associated interstitial lung diseases.

\section{Publication bias}

Publication bias were evaluated with Funnel plots. The funnel plots of any comparisons were symmetrical (Supplementa fig S1-3). Therefore, publication bias was considered unlikely.

\section{Heterogeneity source and sensitivity analysis}

Heterogeneity was observed in the incidence of ILD. Nevertheless, subgroup analyses by ethnicity and classification of ILD reduced the heterogeneity (Table 2-4). Sensitivity analysis performed by excluding studies one by one did not reveal any apparent possible heterogeneity source (data not shown).

\section{Discussion}


To our knowledge, this is the first meta-analysis of the TERT rs2736100 polymorphism and susceptibility to ILD. We found rs2736100_A was significantly associated with ILD in both European and Asian population. The TERT rs2736100 minor allele, rs2736100_C, was lower frequent in Asian population. Subgroup analysis stratified by classification of ILD revealed that the TERT rs $2736100 \mathrm{~A} / \mathrm{C}$ polymorphism was genetic risk factor for IPF, but not for CTD-ILD.

Previous studies demonstrated genes involved in the host-defense, cell-cell adhesion, DNA repair were associated with the incidence of ILD. According to GWAS studies, identified genetic risk foci include MUC5B (rs35705950), TERT (rs2736100), TOLLIP (rs111521887, rs5743894, rs2743890), IL 1RN (rs408392, rs419598), DSP(rs2076295), FAM13A (rs2609255), IL8 (rs4073, rs2227307) and so on [5-7]. The MUC5B rs35705950 has been consider as a common and strong genetic risk factor for ILD in general population [13].

Our study confirmed the TERT rs2736100 polymorphism was a strong risk factor for IPF, while no association was observed in patients with CTDILD. OR for AA vs. CC was higher than that for AC vs. CC (1.77 vs 1.25). In a previous study, the TERT rs 2736100 polymorphism was associated with other ILD but not IPF [14]. In a Chinese population study, no significant difference of the TERT rs2736100 polymorphism was found between IPF and controls [15]. Of note, the sample sizes were small in these two studies.

Ethnic differences of the TERT rs2736100 polymorphism have been analyzed in our study. In a SNP association study in cohorts of Mexican and Korean patients with IPF, after adjusted for MUC5B rs35705950, the TERT rs2736100 polymorphism was associated with IPF in Mexican patients, but not in Korean patients [11]. We found Asian patients had a lower rs2736100_C frequency. For A vs C, AA vs CC, AA + AC vs CC genetic models, OR of Asian ILD patients was higher than that of European group.

In this study, no association of the TERT rs2736100 polymorphism was observed in patients with CTD-ILD. Two studies, involving rheumatoid arthritis (RA) patients and anti-neutrophil cytoplasmic antibody-associated vasculitis (AAV) patients, have been included in this meta-analysis [10, 16]. We noticed that control groups of these studies were patients with CTD. These results might suggest TERT may also participate in pathogenesis of RA or AAV patients without ILD.

The mechanism of the TERT rs2736100 polymorphism in ILD remains unclear. Emerging evidences suggest the incidence of ILD increases with telomere abnormalities. Shorter telomere lengths (TLs) had more frequency in IPF and other ILD (e.g. CTD-ILD and HP). According to previous studies, TLs in AT2 cells of IPF lung were shorter than controls and were associated with total collagen of patients [17]. Shorter blood leukocyte TL was also observed in patients with IIP [18]. The shorten changes of telomere can be induced by mutations in the TERT rs2736100 polymorphism. IPF homozygous for rs2736100_C had longer telomeres than patients homozygous for rs2736100_A [13]. Hence, we suppose the role of the TERT rs2736100 polymorphism in the occurrence and development of ILD might be a result from its effects on telomere length. Furthermore, a luciferase assay study on pulmonary epithelial cells showed the enhancer activity of rs2736100_A was lower than that of rs2736100_C, and was also related with decreased expression of TERT mRNA [19]. Further studies are warranted to clarify the role of the TERT rs2736100 polymorphism in ILD.

This study has several limitations. High heterogeneity existed among the studies. Subgroup analysis according to ethnicity and classification of ILD did not completely reduce heterogeneity, which could be explained by the diverse study design. ILD could be effected by multiple factors, such as age, gender and smoking, so characteristics of study subjects will increase the heterogeneity of our study. The selection of control groups may also influence the heterogeneity. Some studies used healthy people as control groups, while other studies chose patients without ILD, such as asthma or RA. Furthermore, the number of included CTD-ILD studies was small and genotype distribution of rs 2736100 was not available. Therefore, metaanalysis of genetic models could not be performed.

\section{Conclusion}

In conclusion, this study demonstrated the frequency of rs2736100_A was lower in Asian population. We confirmed the TERT rs2736100 A/C polymorphism was a strong risk factor for IPF, but not for CTD-ILD. Whether the incidence of CTD-ILD increases with the TERT rs2736100 A/C polymorphism needs to be evaluated in the future.

\section{Declarations}

\section{Ethics approval and consent to participate}

This study does not involve with ethics as it is a systematic review and meta-analysis.

\section{Consent for publication}

No patient involved.

\section{Availability of data and materials}

The datasets generated during and analysed during the current study are available from the corresponding author on reasonable request. 
All authors do not have any conflicts of interest to disclose.

\section{Funding}

This study is supported by Sichuan Science and Technology Program (2021JDRC0045, 2021YFS0164, 2021YJ0472, 2021JDRC0169), and Clinical Research Incubation Project of West China Hospital, Sichuan University (2019HXFH038).

\section{Authors' contributions}

Xie, Yin and Cui conceived the study, Chen and Cui designed the study forms, and Yin and Xie guided this study. Cui searched the literature; Chen and Ma screened the studies for inclusion and extracted data; Chen and Cui assessed methodological quality; Chen and Cui organized data. All authors drafted and revised the manuscript.

\section{Acknowledgements}

Not applicable

\section{References}

1. McLean-Tooke A, Moore I, Lake F (2019) Idiopathic and immune-related pulmonary fibrosis: diagnostic and therapeutic challenges. Clin Transl Immunology 8:e1086. https://doi.org/10.1002/cti2.1086.

2. Kekevian A, Gershwin ME, Chang C (2014) Diagnosis and classification of idiopathic pulmonary fibrosis. Autoimmun Rev 13:508-512. https://doi.org/10.1016/j.autrev.2014.01.037.

3. Shao T, Shi X, Yang S et al (2021) Interstitial Lung Disease in Connective Tissue Disease: A Common Lesion With Heterogeneous Mechanisms and Treatment Considerations. Front Immunol 12:684699. https://doi.org/0.3389/fimmu.2021.684699.

4. Hamblin M, Prosch H, Vašáková M (2022) Diagnosis, course and management of hypersensitivity pneumonitis. Eur Respir Rev 31:210169. https://doi.org/10.1183/16000617.0169-2021.

5. Diaz de Leon A, Cronkhite JT, Katzenstein AL et al (2010) Telomere lengths, pulmonary fibrosis and telomerase (TERT) mutations. PLoS One 5:e10680. https://doi.org/10.1371/journal.pone.0010680.

6. Newton CA, Batra K, Torrealba J et al (2016) Telomere-related lung fibrosis is diagnostically heterogeneous but uniformly progressive. Eur Respir J 48:1710-1720. https://doi.org/10.1183/13993003.00308-2016.

7. Borie R, Bouvry D, Cottin V et al (2019) Regulator of telomere length 1 (RTEL 1) mutations are associated with heterogeneous pulmonary and extra-pulmonary phenotypes. Eur Respir J 53:1800508. https://doi.org/10.1183/13993003.00508-2018.

8. Fingerlin TE, Murphy E, Zhang W et al (2013) Genome-wide association study identifies multiple susceptibility loci for pulmonary fibrosis. Nat Genet 45:613-620. https://doi.org/10.1038/ng.2609.

9. Mushiroda T, Wattanapokayakit S, Takahashi A et al (2008) A genome-wide association study identifies an association of a common variant in TERT with susceptibility to idiopathic pulmonary fibrosis. J Med Genet 45:654-656. https://doi.org/ 10.1136/jmg.2008.057356.

10. Jönsson E, Ljung L, Norrman E et al (2021) Pulmonary fibrosis in relation to genetic loci in an inception cohort of patients with early rheumatoid arthritis from northern Sweden. Rheumatology (Oxford) 16:1-10. https://doi.org/10.1093/rheumatology/keab441.

11. Peljto AL, Selman M, Kim DS et al (2015) The MUC5B promoter polymorphism is associated with idiopathic pulmonary fibrosis in a Mexican cohort but is rare among Asian ancestries. CHEST 147: 460 - 464. https://doi.org/10.1378/chest.14-0867.

12. Dressen A, Abbas AR, Cabanski C et al (2018) Ramalingam TR, Analysis of protein-altering variants in telomerase genes and their association with MUC5B common variant status in patients with idiopathic pulmonary fibrosis: a candidate gene sequencing study. Lancet Respir Med 6:112. https://doi.org/10.1016/S2213-2600(18)30135-8.

13. Matson SM, Deane KD, Peljto AL et al (2022) MUC5B Prospective Identification of Subclinical Interstitial Lung Disease in a Rheumatoid Arthritis Cohort Is Associated with the Promoter Variant. Am J Respir Crit Care Med 205:473-476. https://doi.org/10.1164/rccm.202109-2087LE.

14. Wei R, Li C, Zhang M et al (2014) Association between MUC5B and TERT polymorphisms and different interstitial lung disease phenotypes. Transl Res 163:494-502. https://doi.org/10.1016/j.trsl.2013.12.006.

15. Wang H, Zhuang Y, Peng $\mathrm{H}$ et al (2019) The relationship between MUC5B promoter, TERT polymorphisms and telomere lengths with radiographic extent and survival in a Chinese IPF cohort. Sci Rep 9:15307. https://doi.org/10.1038/s41598-019-51902-6.

16. Kawasaki A, Namba N, Sada KE et al (2020) Association of TERT and DSP variants with microscopic polyangiitis and myeloperoxidase-ANCA positive vasculitis in a Japanese population: a genetic association study. Arthritis Res Ther 22:246. https://doi.org/ 10.1186/s13075-02002347-0. 
17. Alder JK, Chen JJ, Lancaster L et al (2008) Short telomeres are a risk factor for idiopathic pulmonary fibrosis. Proc Natl Acad Sci USA 105:13051-13056. https://doi.org/10.1073/pnas.0804280105.

18. Dai J, Cai H, Li H et al (2015) Association between telomere length and survival in patients with idiopathic pulmonary fibrosis. Respirology 20:947-952. https://doi.org/ 10.1111/resp.12566.

19. Wei R, Cao L, Pu H et al (2015) TERT Polymorphism rs2736100-C Is Associated with EGFR Mutation-Positive Non-Small Cell Lung Cancer. Clin Cancer Res 21:5173-80. https://doi.org/10.1158/1078-0432.CCR-15-0009.

20. Guzmán-Vargas J, Ambrocio-Ortiz E, Pérez-Rubio G et al (2021) TERT, DSP Differential Genomic Profile in , and Between COPD Patients With Emphysema, IPF, and CPFE Syndrome. Front Med (Lausanne) 8:725144. https://doi.org/10.3389/fmed.2021.725144.

21. Arimura-Omori M, Kiyohara C, Yanagihara T et al(2020) Association between Telomere-Related Polymorphisms and the Risk of IPF and COPD as a Precursor Lesion of Lung Cancer: Findings from the Fukuoka Tobacco-Related Lung Disease (FOLD) Registry. Asian Pac J Cancer Prev 21:667-673. https://doi.org/10.31557/APJCP.2020.21.3.667.

22. Yuan B, Wen X, Li L et al (2020) NAF1 rs4691896 Is Significantly Associated with Coal Workers' Pneumoconiosis in a Chinese Han Population: A Case-Control Study. Med Sci Monit 26:e9187091-e9187096. https://doi.org/10.12659/MSM.918709.

23. Mathai SK, Humphries S, Kropski JA et al (2019) MUC5B variant is associated with visually and quantitatively detected preclinical pulmonary fibrosis. Thorax 74:1-9. https://doi.org/10.1136/thoraxjnl-2018-212430.

\section{Figures}
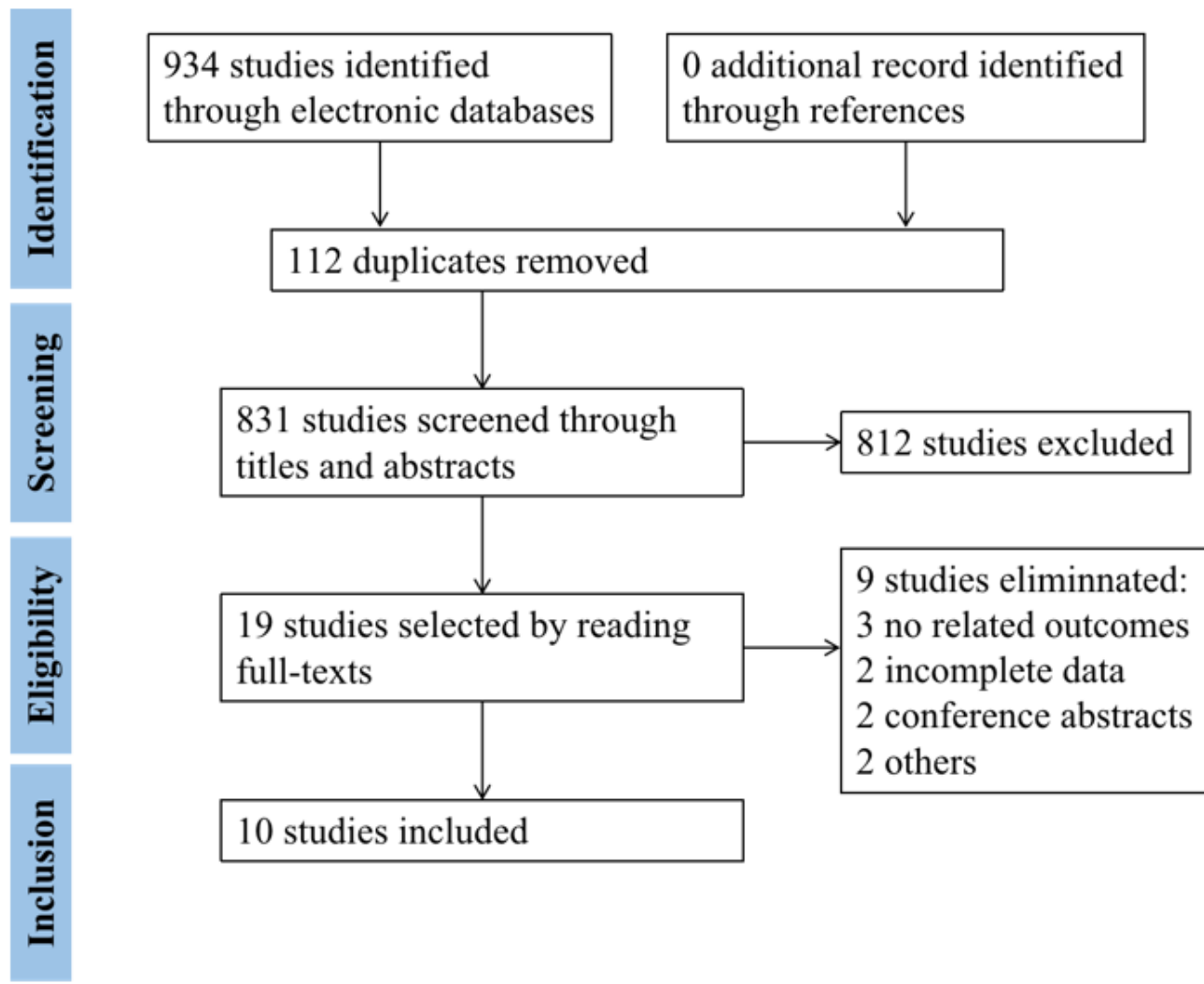

\section{Figure 1}

Study selection flowchart. 


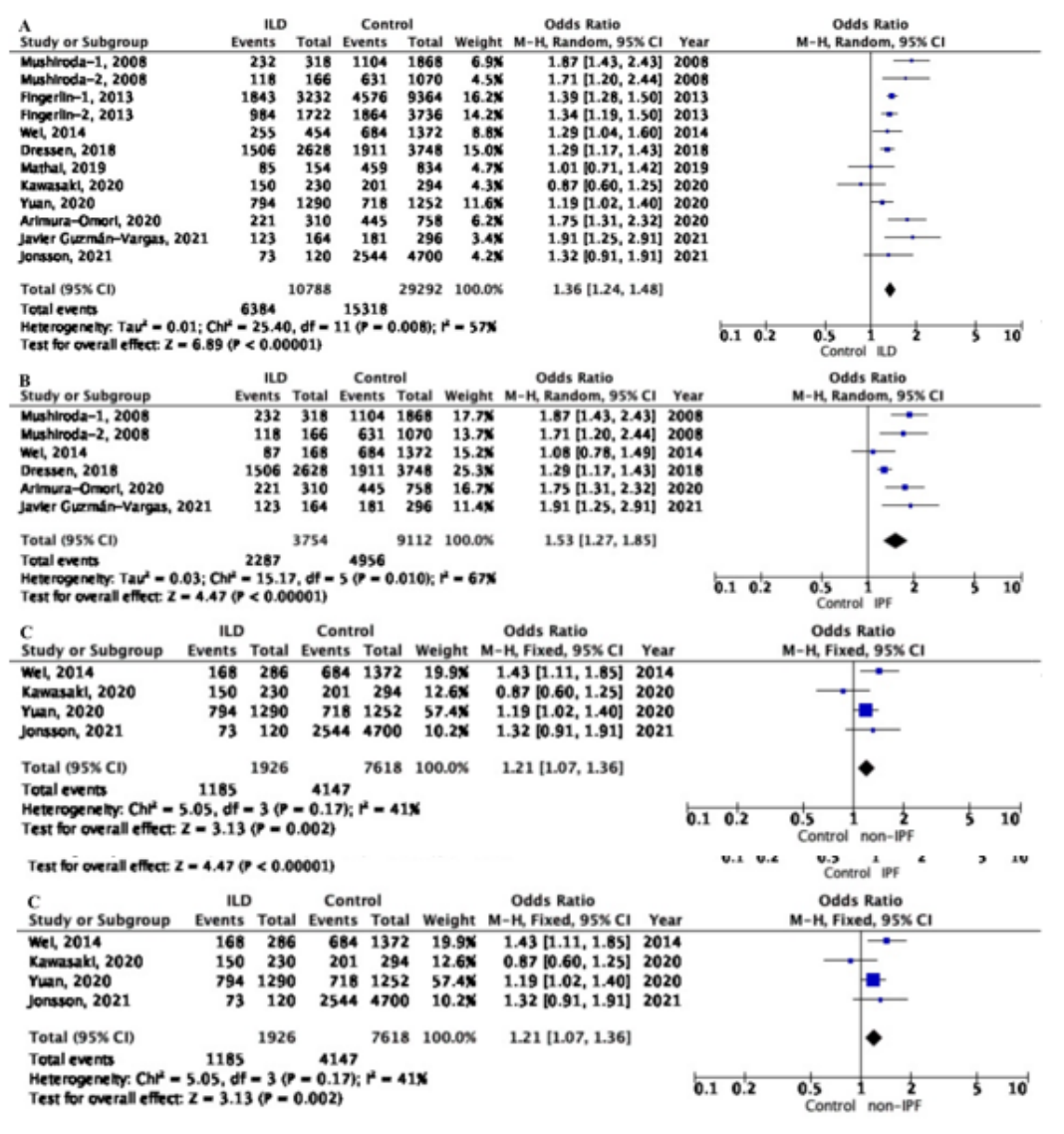

Figure 2

Odds ratios and $95 \%$ confidence intervals from individual studies and pooled data, for association between rs2736100_A and ILD (A), IPF (B) and other ILD (C).

\section{Supplementary Files}

This is a list of supplementary files associated with this preprint. Click to download.

- Supplementaltable1Themethodologicalqualityas.docx

- ildfunnelplot.png

- ipfFunnelplot.png

- nonipfFunnelplot.png 